\title{
IN-SITU MEASUREMENTS OF CHONDROCYTE DEFORMATION UNDER TRANSIENT LOADING
}

\author{
Nadeen O. Chahine 1,2\#, Clark T. Hung 2, Gerard A. Ateshian 1*
}

1 Musculoskeletal Biomechanics Laboratory, 2 Cellular Engineering Laboratory, Department of Biomedical Engineering, Columbia University, New York, NY 10027

\begin{abstract}
Chondrocytes are responsible for the elaboration and maintenance of the extracellular (EC) matrix in articular cartilage, and previous studies have demonstrated that mechanical loading modulates the biosynthetic response of chondrocytes in cartilage explants. The goal of this study is to investigate the deformation behaviour of the chondrocyte and its microenvironment under transient loading, in order to address the relationship between the applied dynamic deformation and cellular strain. In situ strain measurements were performed on cells in the middle (MZ) zone at early time points during ramp loading and at equilibrium. In this study, we characterized the behaviour of cartilage at the zonal and cellular levels under compressive loading using digital image analysis on miniature samples tested in a custom microscopy-based loading device. The experimental results indicate that significant strain amplification occurs in the microenvironment of the cell, with the minimum (compressive) principal strain found to be nearly $7 \mathrm{X}$ higher in the intracellular region (IC), and $\sim 5 \mathrm{X}$ higher in the pericellular (PC) matrix than in the EC matrix at peak ramp. A similar strain amplification mechanism was observed in the maximum (tensile) principal strain, and this behaviour persisted even after equilibrium was reached. The experimental results of this study were interpreted in the context of a finite element model of chondrocyte deformation, which modelled the cell as a homogeneous gel, possessing either a spherical or ellipsoidal geometry, surrounded by a semi permeable membrane, and accounted for the presence of a PC matrix. The results of the FEA demonstrate significant strain amplification mechanism in the IC region, greater than had previously been suggested in earlier computational studies of cell-EC matrix interactions. Based on the FEA, this outcome is understood to result from the large disparity between EC matrix and intracellular properties. The results of this study suggest that mechanotransduction of chondrocytes may be significantly mediated by this strain amplification mechanism during loading.
\end{abstract}

Keywords: Cellular deformation, cartilage, cell-matrix interaction.

\# Current Affiliation: Center for Micro and Nano Technology, Lawrence Livermore National Laboratory, Livermore, CA 94550
* Address for correspondence:

Gerard A. Ateshian

Department of Mechanical Engineering, Columbia University

SW Mudd, 500 West 120th Street, Mail Code 4703

New York, NY 10027

Telephone Number: 212-854-8602

FAX Number: 212-854-3304

E-mail: ateshian@columbia.edu

\section{Introduction}

Chondrocytes, the sparsely distributed cells found in articular cartilage, are responsible for the elaboration of extracellular matrix components of cartilage, such as Type II collagen and proteoglycans (PG). Previous studies have demonstrated the efficacy of mechanical loading on the normal maintenance of articular cartilage in vivo (Palmoski et al., 1979; Setton et al., 1995) and in vitro (Gray et al., 1988; Sah et al., 1989; Sah et al., 1991; Guilak et al., 1994; Valhmu et al., 1998; Buschmann et al., 1999; Wong et al., 1999), indicating that mechanical loading modulates the biosynthetic response of chondrocytes in cartilage explants. In vitro studies of articular cartilage metabolism have demonstrated that static loading as well as loading below a characteristic frequency of $0.001 \mathrm{~Hz}$ leads to biosynthetic inhibition, whereas dynamic loading stimulates tissue synthesis (Palmoski and Brandt, 1984; Sah et al., 1989; Guilak et al., 1994; Kim et al., 1994; Guilak et al., 1997). For example, static compressive loading has been shown to inhibit chondrocyte PG and protein synthesis, as well as reduce the release of PG from the extra-cellular (EC) matrix (Palmoski and Brandt, 1984; Gray et al., 1988; Guilak et al., 1994). Conversely, dynamic loading has been shown to generally stimulate the biosynthetic response of chondrocytes, though these responses are strongly dependent on magnitude and frequency of the applied load (Palmoski and Brandt, 1984; Sah et al., 1989; Sah et al., 1991; Buschmann et al., 1999). Despite numerous studies demonstrating the responsiveness of chondrocytes to loading, the complexity in the mechanical response and properties of cartilage have confounded the examination of how these mechanical signals are conveyed from the tissue level down to the cellular level.

Articular cartilage possesses complex mechanical properties such as depth-dependent inhomogeneity and tension-compression nonlinearity (Kempson et al., 1968; Woo et al., 1976; Akizuki et al., 1986; Schinagl et al., 1997; Wang et al., 2002a). The ultrastructure and 
alignment of matrix molecules such as collagen and PG also result in directional dependence in the matrix mechanical properties, known as anisotropy (Kempson et al., 1968; Akizuki et al., 1986; Wang et al., 2003). Additionally, the presence of proteoglycans in the tissue attracts water into the cartilage matrix, resulting in a dense hydrated environment (Maroudas, 1979; Maroudas and Bannon, 1981). When loaded, the tension in the collagen fibres limits the lateral expansion of the tissue, and therefore the interstitial fluid pressurizes and experiences resistance to flow out of the tissue. This fluid pressurization increases the tissue's ability to bear load by allowing the applied load to be sustained by both the extracellular matrix as well as the pressurized fluid (Soltz and Ateshian, 2000). Consequently, cells present in cartilage during loading may be exposed to a variety of mechanical signals - such as compressive and tensile stresses and strain, fluid flow, hydrostatic pressure, osmotic pressure and ionic gradients - which may influence the cell's biosynthetic response in situ.

In light of the mechanical complexity of the cartilage matrix, several studies have examined the deformational responses of chondrocytes using theoretical and numerical analyses (Wu et al., 1999; Guilak, 2000; Wu and Herzog, 2000; Guilak et al., 2002). In the studies of Guilak et al., a multi-scale finite element model was developed of the chondrocyte as a spheroidal inclusion embedded within the extracellular matrix of a cartilage explant, and the tissue was assumed to obey a homogeneous, isotropic constitutive relation. Results indicate that the mechanical environment at the cellular level was significantly varied from the mechanical environment at the macroscopic level. Additionally, the presence of a pericellular matrix surrounding the chondrocyte was also found to modulate the response of the cell to mechanical loading (Guilak and Mow, 2000; Guilak et al., 2002). Moreover, more recent studies have shown that tissue behaviour, particularly the strain and stress, to be considerably different when modelling cartilage as a homogeneous versus inhomogeneous tissue (e.g., depth-dependent variation in stiffness and/or fixed charge density) (Wang et al., 2001; Wang et al., 2002b). Indeed, a study on cellular microenvironment in intervertebral disk (IVD) has shown that IVD anisotropy has major implication on the morphology and mechanical environment of cells in the annulus fibrosus versus those in the nucleus pulposus (Baer et al., 2003).

The deformation of chondrocytes has been previously measured, generally under static deformation, in native cartilage (Guilak 1995; Guilak et al., 1995; Guilak et al., 1999; Guilak, 2000; Clark et al., 2003) and in other scaffold materials, e.g. agarose ( Lee and Bader, 1995; Knight et al., 2002). These studies investigated the effect of static loading on cell morphology, deformation index (aspect ratio of cell), cross sectional area and cell volume. In native cartilage, it has been shown that chondrocytes and their nuclei undergo significant changes in shape and volume under static loading, and that the chondrocytes respond differently based on their location through the depth of the tissue (Guilak, 1995; Guilak et al., 1995). For chondrocytes cultured in agarose hydrogels, it was found that matrix elaborated by the cells markedly influenced levels of cell deformation; where an increase in matrix elaboration led to a decrease in cell deformation under static loading (Lee and Bader, 1995).

In contrast to static loading, the deformation of chondrocytes under physiological, dynamic loading has not been widely investigated. A study by Knight et al. demonstrated that chondrocytes seeded in agarose gels deform from a spherical morphology to an oblate ellipsoid morphology under both static and trapezoidal cyclic deformation, applied at $0.3 \mathrm{~Hz}$ (Knight et al., 1998). However, no study to date has investigated the deformational behaviour of chondrocytes in cartilage under dynamic loading.

Therefore, the goal of this study is to investigate the deformation behaviour of the chondrocyte and its microenvironment under transient loading, in order to address the relationship between the applied dynamic deformation and cellular strain. In situ strain measurements are performed in this study on cells in the middle (MZ) zone at early time points during ramp loading and at equilibrium, in unconfined compression. In significant respects, unconfined compression is representative of physiological loading conditions in joints because it subjects cartilage to compression in the thickness direction and tension in the plane tangential to the articular surfaces, similarly to physiological joint contact loading; it also pressurizes the interstitial fluid uniformly through the thickness, as also found for contact loading (Park et al., 2003). In this study, we characterize the behaviour of cartilage at the zonal and cellular levels under compressive loading using digital image analysis on miniature samples tested in custom microscopy loading devices. Furthermore, the cellular deformation results of this study are interpreted in the context of a finite element model of chondrocyte deformation, based on the framework introduced by Guilak et al. (Guilak and Mow, 2000).

\section{Materials and Methods}

\section{Sample Preparation}

Full thickness osteochondral plugs (Ø 4mm) were harvested from the carpometacarpal joint of 3-4 month old calves under sterile conditions and cultured in high glucose Dulbecco's Modified Essential Medium (DMEM) supplemented with $1 \%$ ITS, $100 \mathrm{nM}$ dexamethasone, $50 \mu \mathrm{g} /$ $\mathrm{ml}$ of L-Proline, $100 \mu \mathrm{g} / \mathrm{ml}$ sodium pyruvate, and antibiotics (penicillin, streptomycin). These disks were cultured in a humidified incubator at $37^{\circ} \mathrm{C}$ and $5 \% \mathrm{CO} 2$ with daily supplementation of ascorbic acid. All explants were allowed to acclimate to the culture environment for 2 days prior to experimentation. On the day of testing, all subchondral bone was removed using a custom cutting device. A smaller cylindrical full thickness sample $(\varnothing 2 \mathrm{~mm}$ x $0.71 \pm 0.16 \mathrm{~mm}$ thick) was prepared from the centre of the disk, and cut diametrically to create 2 semi cylindrical specimens. 

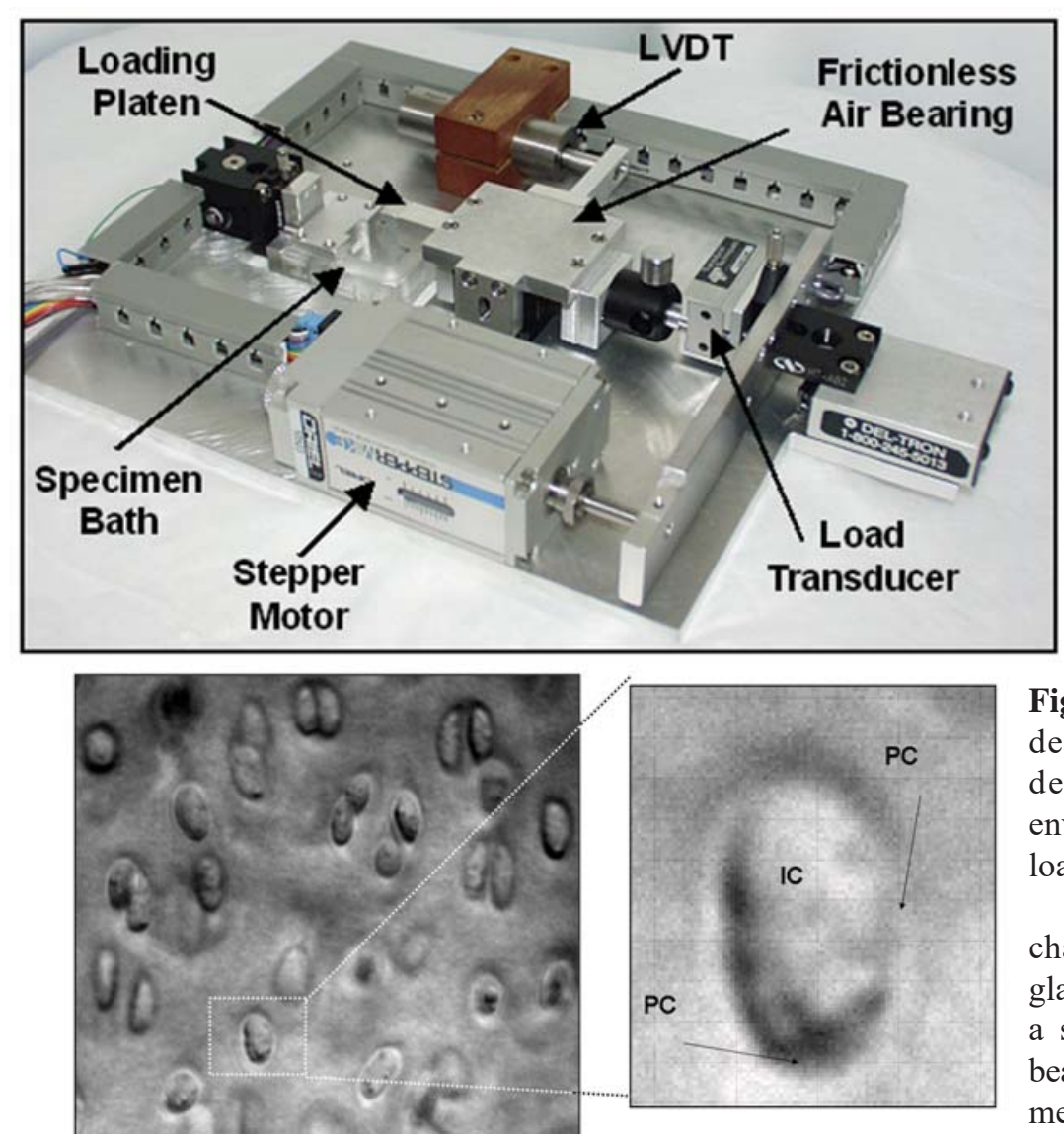

Figure 1: Automated microscopy loading device utilized in measuring the cell deformation behavior in the microenvironment of chondrocytes under ramp loading.

Samples are loaded into the specimen chamber and are compressed between 2 glass platens. Deformation is actuated with a stepper motor and a frictionless air bearing. Resulting load and deformation are measured using the load cell and LVDT.

Study 1: Local deformation of cell microenvironment Semi-cylindrical samples ( $\varnothing 2 \mathrm{~mm} \times 0.71 \pm 0.16 \mathrm{~mm}$ thick; $\mathrm{N}=5$ ) were mounted in a custom-designed computercontrolled motorized loading apparatus (Fig. 1), fitted with a stepper micrometer for displacement actuation (Model 18500, Oriel Instruments, Stratford, CT), LVDT for displacement measurement (Model HR 100, Schaevitz Sensors, Hampton, VA), and load cell for load measurement. The apparatus was mounted on the stage of an inverted microscope (IX70, Olympus, Melville, NY), motorized to provide $\mathrm{X}-\mathrm{Y}$ displacement via computer control, using the LabView data control and acquisition software (National Instruments, Austin, TX). Images were acquired with a MicroMax 5-MHz interline transfer chip camera (Princeton Instruments, Trenton, NJ, 1300'1030 pixels) and Metamorph Imaging system (Universal Imaging, West Chester, PA) having the capability of video rate acquisition (30 frames per second). The cartilage sample, immersed in PBS, was sandwiched between two microscope glass slides ( $1 \mathrm{~mm}$ thick) to prevent out of plane deformation during loading. A tare compression ( $2 \%$ strain) was initially applied, and allowed to equilibrate for 30 minutes. The specimens were then loaded with a ramp displacement representing $2 \%$ strain, applied at $1 \mu \mathrm{m} / \mathrm{sec}$. High magnification (40X; $0.16 \mu \mathrm{m} / \mathrm{pixel})$ images of the samples were acquired during the ramp loading, using a digital camera at a rate of $\sim 2$ frames/second (MicroMax camera, Princeton Instruments, Trenton, NJ). After 30 minutes of equilibration, images of the sample crosssection were also acquired. Images of the chondrocytes and surrounding tissue were acquired using a transmitted light source and optical contrast filter on an inverted microscope in order to provide sufficient texture for strain analysis (Fig. 1). Imaging and strain analysis of the chondrocytes were performed in the median plane bisecting the centre of the cell. This mid plane was qualitatively identified as the one in which the cell cross section was maximized.

Digital image correlation (DIC), tracking the displacement of the cells during the deformation, was performed using VIC-2D (Correlated Solutions, West Columbia, SC). In DIC, texture and patterns inherently created by the cell and the EC matrix are tracked during loading in order to calculate the resulting displacement in the tissue. The displacement analysis is typically carried out by tracking the motion of a subset of pixels within a larger field of view. The DIC analysis employed in this study is conceptually similar to that previously used to study the depth-dependent properties of cartilage (e.g., (Schinagl et al., 1996; Wang et al., 2002a)), though the software and algorithms employed are different. In this analysis, a DIC subset of 29 pixels was used with a step size of 5 pixels, to track the displacement of 2 to 4 regions of interest (ROIs) within the midzone of each cartilage specimen. Each ROI consisted of a single cell with its surrounding pericellular matrix (PC, Fig. 1). No chondrons containing more than one cell were utilized in this analysis. The strain was calculated from the derivative of the displacement field, calculated locally over a window size of 7 neighbouring points. 
The quality of the strain analysis is inherently dependent on the ability of the algorithm to track subset patterns between images; consequently analysis in this study is performed on sequential images acquired during the ramping phase of the experiment. The total strain is calculated as the sum of the average strains between the first ( $0-1 \%$ deformation) and second (1 - 2\%) increment. In this study, we report the minimum $\left(e_{1}\right)$ and maximum $\left(e_{2}\right)$ principal strains, averaged over 10 to 12 points from within the intracellular space (IC), and in the surrounding pericellular matrix (PC). In order to validate the quality of the strain analysis under such small deformation (i.e. $<1 \%$ increments), simulated deformations were applied to typical images from this experiment and analyzed using the same DIC technique and parameters used with the loaded images. Simulated compressive deformation of 0.5 , 1.0 and $2.0 \%$ strain were applied either (1) in the axial direction of loading (using linear interpolation of image greyscale), (2) in the lateral direction to loading (linear interpolation), and (3) simultaneously to both the axial and lateral directions (bilinear interpolation). The simulated image deformation was performed using Matlab (MathWorks Inc, Natick, MA). The resulting images were analyzed using VIC-2D as described above. The resulting average $e_{1}$ and $e_{2}$ were calculated for 1 ROI from 3 samples.

\section{Study 2: Finite element analysis of cell deformation}

To investigate the local deformation behaviour of chondrocytes in situ, a finite element analysis conceptually similar to the study of Guilak and Mow (Guilak and Mow, 2000) was performed, applying the modifications recently described by Ateshian et al. (2007). A multiscale analysis is performed whereby the chondrocyte is embedded in the centre of a cylindrical cartilage disk. The sample is loaded in unconfined compression between 2 impermeable platens on the top and bottom surfaces. The stress relaxation response of the disk is obtained from the closed form solution of an axisymmetric analysis of a biphasic material (Mow et al., 1980). The resulting solutions for the displacements $u_{r}(r, t)$ and $u_{z}(z, t)$ and pressure $p(r, t)$ were applied as boundary conditions on a finite element mesh of the chondrocyte and its surrounding EC matrix, where

$$
\begin{gathered}
\frac{u_{r}(r, t)}{r_{0}}=-\frac{\dot{\varepsilon}_{0}}{2}\left[\left(\frac{2 \lambda_{2}}{H_{+A}+\lambda_{2}}\right) \frac{r}{r_{0}} t-\sum_{n=1}^{\infty} \frac{2\left(H_{+A}-\lambda_{2}\right)}{\gamma_{n}\left[\left(H_{+A}+\lambda_{2}\right) J_{0}\left(\gamma_{n}\right)-\gamma_{n} H_{+A} J_{1}\left(\gamma_{n}\right)\right]}\right. \\
\left.J_{1}\left(\gamma_{n} \frac{r}{r_{0}}\right)\left(\frac{r_{0}^{2}}{H_{+A} k \gamma_{n}^{2}}\right)\left(1-\exp \left(-\frac{H_{+A} k}{r_{0}^{2}} \gamma_{n}^{2} t\right)\right)\right](1) \\
\frac{u_{z}(z, t)}{h}=\dot{\varepsilon}_{0} t \frac{z}{h} \\
p(r, t)=\dot{\varepsilon}_{0} H_{+A}\left[\left(\frac{2 \lambda_{2}}{H_{+A}+\lambda_{2}}\right) t-\sum_{n=1}^{\infty} \frac{\left(H_{+A}-\lambda_{2}\right)}{\left(H_{+A}+\lambda_{2}\right) J_{0}\left(\gamma_{n}\right)-\gamma_{n} H_{+A} J_{1}\left(\gamma_{n}\right)}\right. \\
\left.J_{0}\left(\gamma_{n} \frac{r}{r_{0}}\right)\left(\frac{r_{0}^{2}}{H_{+A} k \gamma_{n}^{2}}\right)\left(1-\exp \left[-\frac{H_{+A} k}{r_{0}^{2}} \gamma_{n}^{2} t\right]\right)\right]
\end{gathered}
$$

In these expressions, $r_{0}$ is the radius of the cartilage sample and $h$ is its thickness, $\dot{\varepsilon}_{0}$ is the compressive strain rate, $H_{+A}$ is the tensile aggregate modulus and $\lambda_{2}$ is the off-diagonal modulus of the EC matrix (Soltz and Ateshian, 2000), and $k$ is its hydraulic permeability.

For the purpose of the current analysis the chondrocyte is modelled as a homogeneous gel, representing the protoplasm (cytoplasm, cytoskeleton, and all enclosed organelles), surrounded by a semi permeable membrane. The EC matrix is modelled as a biphasic disk possessing tension-compression nonlinearity in cubic symmetry (Chahine et al., 2004), with a higher tensile stiffness ( $H_{+A}$ $=2 \mathrm{MPa})$ than compressive stiffness $\left(H_{-\mathrm{A}}=0.4 \mathrm{MPa}\right)$, with $\lambda_{2}=0$, and the permeability of the EC matrix was $k=5 \times 10^{-15} \mathrm{~m}^{4} / \mathrm{N} \cdot \mathrm{s}$. The cell protoplasm is also modelled as a biphasic matrix, possessing a Young's modulus of $E_{Y}$ $=1 \mathrm{kPa}$ and Poisson's ratio $v=0.3$ (Trickey et al., 2000; Trickey et al., 2006), and permeability ( $k$ ) equal to that of the EC matrix. The semi-permeable membrane provided resistance to flow, with a hydraulic conductivity of $L_{p}=3 \times 10^{-14} \mathrm{~m}^{3} / \mathrm{N} \cdot \mathrm{s}$ (Xu et al., 2003), and the modulus of the membrane was taken to be the same as the matrix. In some cases, the pericellular matrix was also modelled, possessing $E_{Y}=40 \mathrm{kPa}, \quad v=0.05$, and $k=4 \times 10^{-17} \mathrm{~m}^{4} / \mathrm{N} \cdot \mathrm{s}$ (Alexopoulos et al., 2005b).

The effect of chondrocyte geometry on local cell deformation was investigated. The chondrocyte was modelled either as an idealized sphere, with a diameter of $10 \mu \mathrm{m}$, or based on the in situ geometry as determined from digitization of the images acquired in Study 1. The in situ geometry replicates the cell as an approximate prolate ellipsoid with major and minor diameters of 12 and $8 \mu \mathrm{m}$, respectively, where the major diameter is in a direction approximately perpendicular to the direction of loading. When modelled, the PC matrix was represented as 3- $\mu \mathrm{m}$ thick layer surrounding the cell membrane (Youn et al., 2006). The local EC region modelled around the cell extends $50 \mu \mathrm{m}$ along the axial and radial directions, with the cell centred in this ROI. Because of numerical stability considerations the true thickness of the membrane (on the order of $10 \mathrm{~nm}$ ) was not modelled. Instead, a layer of thin elements $\left(h_{m}=50 \mathrm{~nm}\right)$ was used to represent the membrane, and the hydraulic permeability of these elements $\left(k_{m}=1.5 \times 10^{-21} \mathrm{~m}^{4} / \mathrm{Ns}\right)$ was modified accordingly to represent the hydraulic conductivity of the membrane ( $\left.L_{p}=k_{m} / h_{m}\right)$. This approach has been previously used and validated (Ateshian et al., 2007).

The finite element mesh consists of 8-node quadrilateral elements, and was created using commercial software (I-deas NX Series v.11, UGS Corp., Plano, TX), with either 1766 or 2803 nodes and 561 or 904 elements used to create the meshes for the spherical or ellipsoidal cell models, respectively. The analysis is performed using a custom-written finite element code for biphasic problems (Krishnan et al., 2003), which employs a displacementpressure $(u-p)$ formulation (Wayne et al., 1991; Almeida and Spilker, 1997). Results are plotted using commercial software (Tecplot 10.0, Tecplot Inc., Bellevue, WA).

\section{Statistical Analysis}

Statistical analysis was performed on experimental measures of $e_{1}$ and $e_{2}$ using an ANOVA, testing for the effect of loading time (ramp vs. equilibrium). A repeated measure was introduced into the analysis to account for the differences in the cell regions (IC vs. PC). An LSD 
Table 1: Strain results of the simulated compressive deformations applied along the (a) axial direction, (b) lateral direction or (c) bidirectionally, along the axial and lateral directions. The magnitudes of the simulated deformation are $-0.005,-0.01$ and -0.02 . Each result represents mean \pm standard deviation $(n=3$ per group).

\begin{tabular}{c|c|c|c|c|c|c|} 
& \multicolumn{2}{|c|}{ Axial } & \multicolumn{2}{c|}{ Lateral } & \multicolumn{2}{c|}{ Axial \& Lateral } \\
\cline { 2 - 7 } & $e_{1}=\varepsilon$ & $e_{2}=0$ & $e_{1}=\varepsilon$ & $e_{2}=0$ & $e_{1}=\varepsilon$ & $e_{2}=\varepsilon$ \\
\hline \multirow{2}{*}{$\varepsilon=-0.005$} & $-0.0044 \pm$ & $1.05 \mathrm{E}-05 \pm$ & $-0.0052 \pm$ & $2.64 \mathrm{E}-05 \pm$ & $-0.0043 \pm$ & $-0.0053 \pm$ \\
& 0.0003 & $4.21 \mathrm{E}-05$ & 0.0001 & $1.01 \mathrm{E}-05$ & 0.0002 & 0.0002 \\
\hline \multirow{2}{*}{$\varepsilon=-0.01$} & $-0.0098 \pm$ & $-1.32 \mathrm{E}-06 \pm$ & $-0.0093 \pm$ & $2.72 \mathrm{E}-05 \pm$ & $-0.0090 \pm$ & $-0.0101 \pm$ \\
& 0.0005 & $4.34 \mathrm{E}-05$ & 0.0001 & $4.44 \mathrm{E}-05$ & 0.0001 & 0.0003 \\
\hline \multirow{2}{*}{$\varepsilon=-0.02$} & $-0.0195 \pm$ & $7.83 \mathrm{E}-06 \pm$ & $-0.0193 \pm$ & $-7.72 \mathrm{E}-06 \pm$ & $-0.0190 \pm$ & $-0.0200 \pm$ \\
& 0.0003 & $3.15 \mathrm{E}-05$ & 0.00003 & $8.33 \mathrm{E}-05$ & 0.0001 & 0.0001 \\
\hline
\end{tabular}
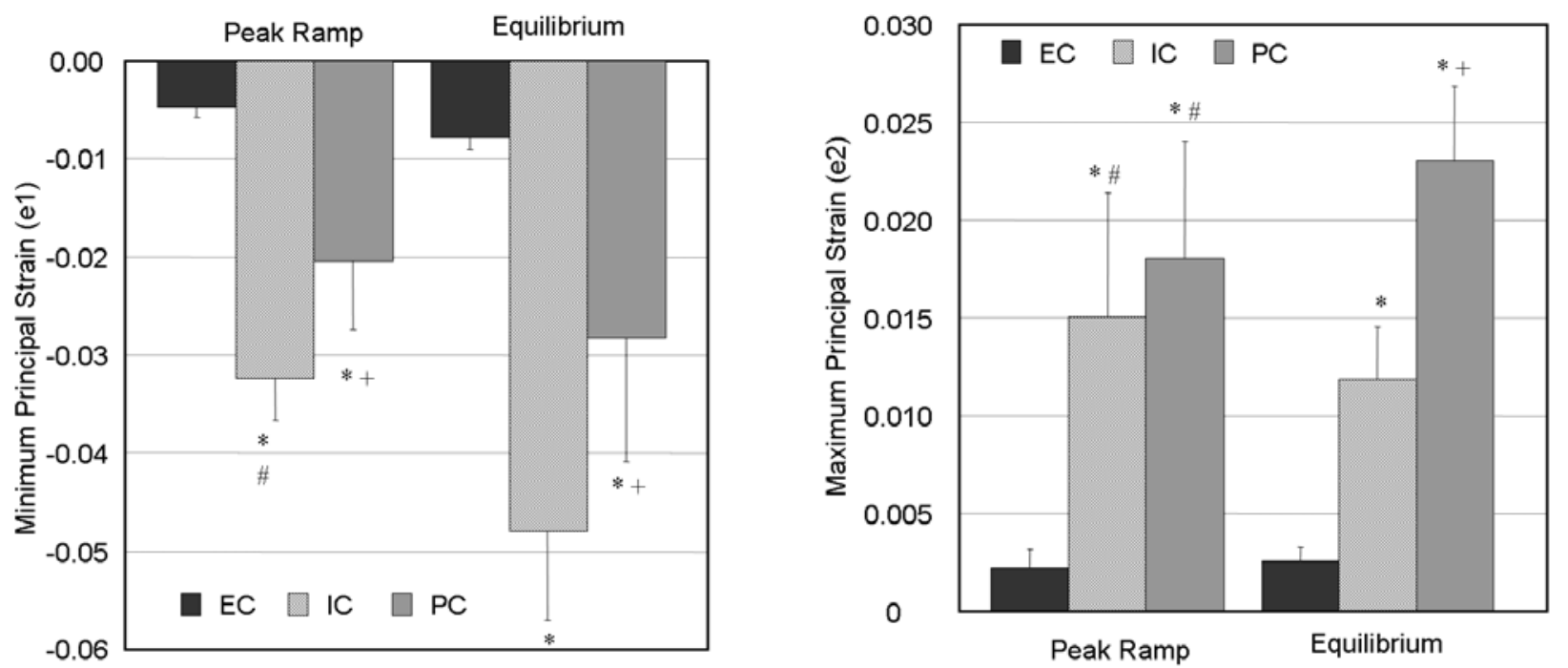

Figure 2: Average minimum $\left(e_{1}\right)$ and maximum $\left(e_{2}\right)$ principal strain measured in $\mathrm{MZ}$ chondrocytes at end of ramp loading ( $2 \%$ platen-to-platen) or at equilibrium. Strain microenvironment is reported in the intracellular (IC) and pericellular (PC) regions of chondrocytes. * indicates significance relative to matrix strain, + indicates significance relative to $\mathrm{PC}$, \# indicates significance relative to corresponding group at equilibrium.

post hoc test was applied, with $p<0.05$ considered significant.

\section{Results}

In order to assess the accuracy of the imaging and analysis technique, the average minimum $\left(e_{1}\right)$ and maximum $\left(e_{2}\right)$ principal strains for simulated deformations of -0.005 , 0.01 , and -0.02 are presented in Table 1 . The average $e_{1}$ measured for a simulated deformation of -0.005 was found to be $-0.0044 \pm 0.0003$ and $-0.0052 \pm 0.0001$ when applied in the axial and lateral directions, respectively (Table 1). The corresponding perpendicular deformation (which was imposed to be zero) was calculated to be $1.05 \times 10^{-5} \pm$ $4.21 \times 10^{-5}$ and $2.64 \times 10^{-5} \pm 1.01 \times 10^{-5}$, respectively. With increasing deformation, the accuracy of the DIC increased. The average $e_{1}$ measured at an applied deformation of -0.02 was found to be $-0.0195 \pm 0.0003$ and $-0.0193 \pm$ 0.00003 in the axial and lateral directions, respectively. Comparable accuracy was measured when deformation was applied bidirectionally to the axial and lateral directions simultaneously (Table 1). Consequently, the accuracy of the DIC technique used in this study was found to be within $\sim 10 \%$ of the applied deformation for the smallest strains $(-0.005)$, with the accuracy improving to be within $\sim 2$ to $3 \%$ of the applied deformation of at larger strain magnitude $(-0.02)$. 

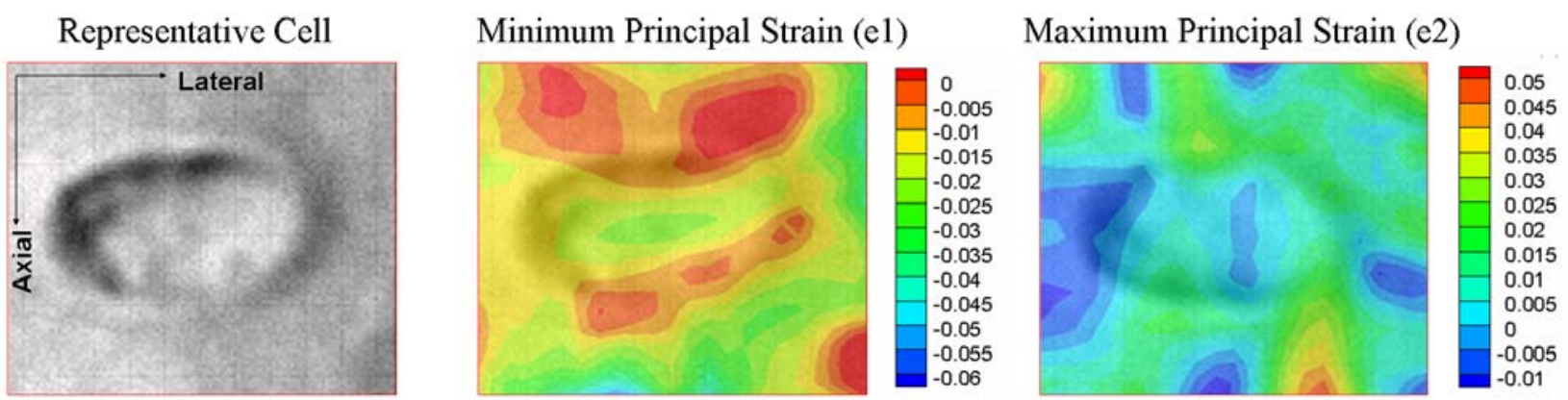

Figure 3: Representative local deformation in and around a chondrocyte from the $\mathrm{MZ}$ of cartilage. Minimum $\left(e_{1}\right)$ and maximum principal $\left(e_{2}\right)$ strains are plotted at the midpoint of the ramp (i.e. at $1 \%$ platen-to-platen strain).
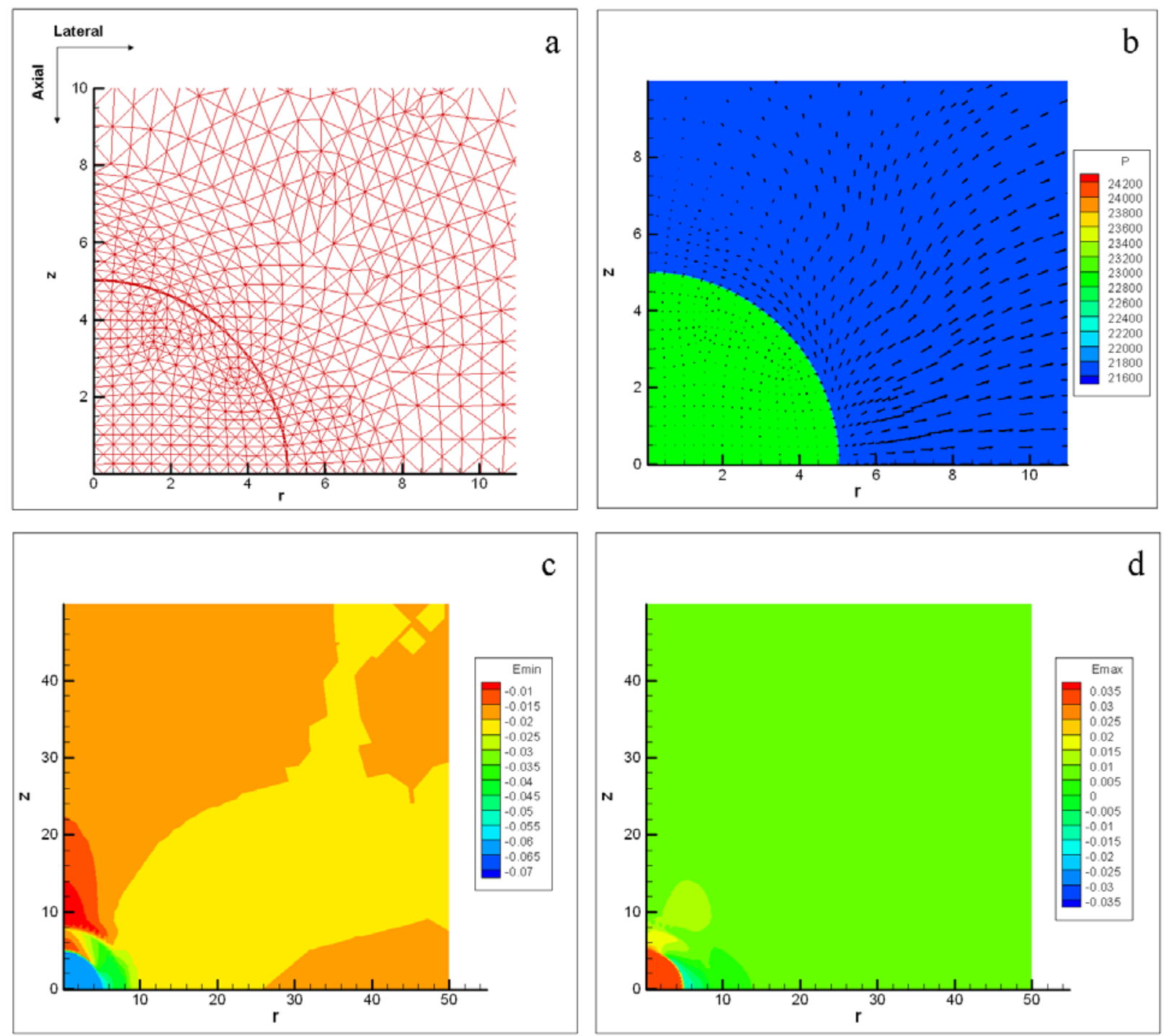

Figure 4: Results of finite element model, examining the fluid pressure and local cellular deformation around a spherical chondrocyte. A $2 \%$ platen-to-platen deformation ramp deformation was applied in the y-direction. (a) Spherical mesh used in analysis, (b) hydrostatic pressure inside and outside the cell - vectors indicate direction of fluid flow, (c) minimum principal strain and (d) maximum principal strains. 

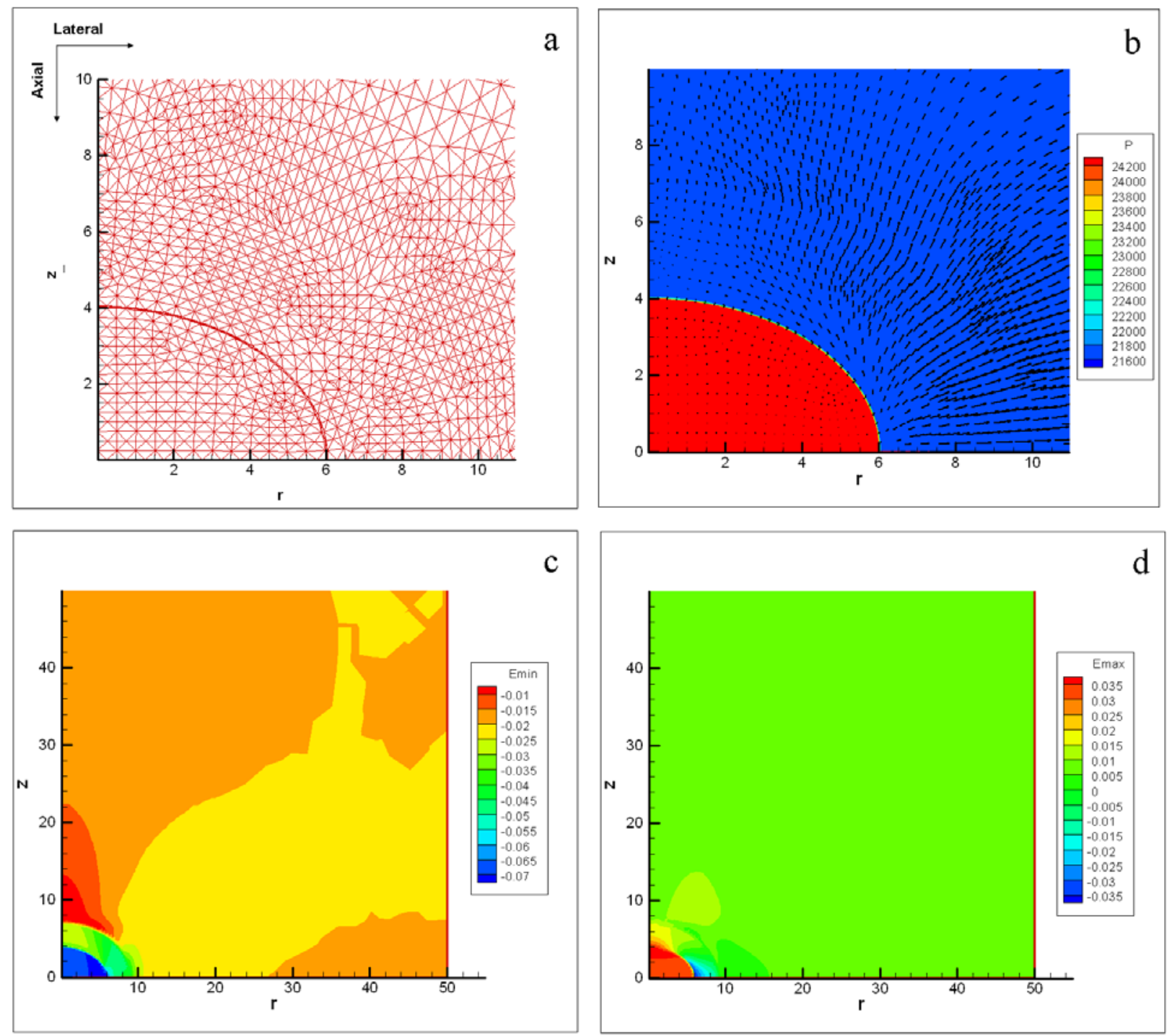

Figure 5: Results of finite element model, examining the fluid pressure and local cellular deformation around an ellipsoidal chondrocyte. All results are plotted at peak ramp deformation of $2 \%$ platen-to-platen deformation. (a) Elliptical mesh used in analysis was determined from the images of cells acquired in Study 2, (b) hydrostatic pressure inside and outside the cell - vectors indicate direction of fluid flow, (c) minimum principal strain and (d) maximum principal strains.

Study 1: Local deformation of cell microenvironment The average minimum $\left(e_{1}\right)$ and maximum $\left(e_{2}\right)$ principal strains are reported in Fig. 2 for cells loaded in situ in mid zone cartilage. The minimum strain $\left(e_{1}\right)$ was found to be negative, thus representing primarily the deformation in the loading direction. A nominal $2 \%$ platen-to-platen ramp deformation resulted in a minimum principal strain of $0.47 \% \pm 0.11 \%$ at the end of ramp (i.e. $e_{1}=-0.0047 \pm$ $0.0011)$. This tissue-level deformation resulted in a significantly greater minimum principal strain in the intracellular region of chondrocytes $\left(e_{1}=-0.0324 \pm 0.0043\right.$, $p<0.05$ ). Additionally during ramp loading, the $\mathrm{PC}$ matrix was exposed to a compressive strain of $-0.0205 \pm 0.0069$, a magnitude significantly smaller than measured in the IC $(p<0.05)$. A representative strain contour measured under transient ramp loading is presented in Fig. 3.

At equilibrium, the magnitude of tissue strain was greater than seen under ramp loading, reaching $-0.0079 \pm$
0.0012 . The IC regions were also exposed to a significantly greater magnitude of compressive strain than during ramp loading $\left(e_{1}=-0.0480 \pm 0.0089, p<0.03\right.$, Fig. 2$)$. However, the minimum principal strain in the $\mathrm{PC}$ region was found to be comparable to that under ramp loading $\left(e_{1}=-0.0283\right.$ $\pm 0.013, p>0.9$ ).

The maximum principal strain $\left(e_{2}\right)$ was found to be positive, representing primarily the deformation in a direction perpendicular to the loading direction (Fig. 2). Thus the axial compression resulted in a lateral tensile strain of $0.22 \% \pm 0.10 \%$ in the tissue matrix by the end of the loading ramp (i.e. $e_{2}=0.0022 \pm 0.0010$ ). The magnitude of $e_{2}$ was found to be comparable in the IC and PC regions during ramp loading $(0.0150 \pm 0.0064$ in IC versus 0.0180 \pm 0.0060 in $\mathrm{PC}$ matrix, $p>0.8)$. After equilibrium was reached, the tissue matrix was exposed to a lateral strain of $0.0026 \pm 0.0007$, which was comparable to the lateral strain during ramp loading. However, $e_{2}$ measured in the 

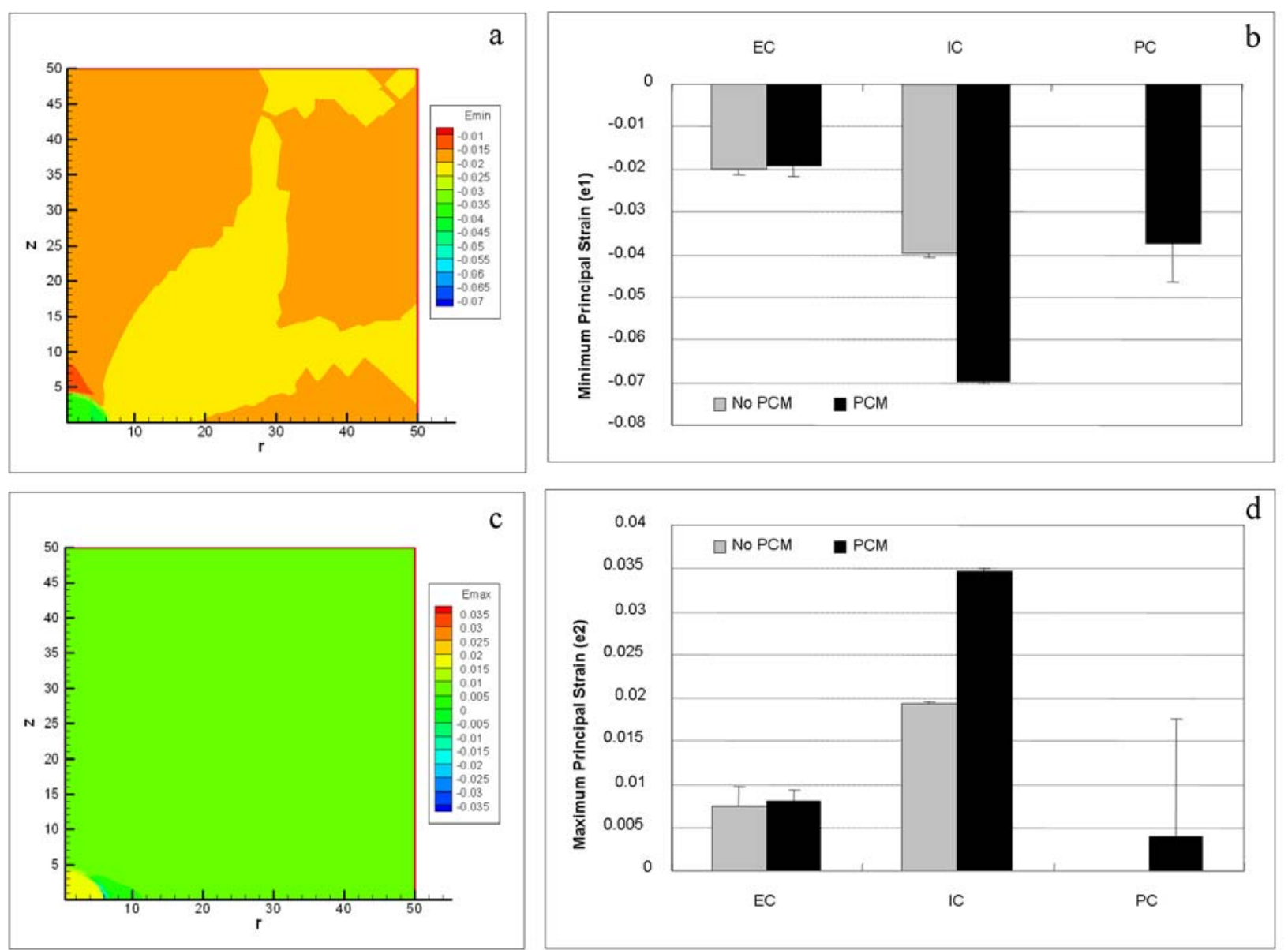

Figure 6: Role of PC matrix on the strain amplification mechanism for ellipsoidal cell. (a) Representative contour plot of the minimum principal strain in the absence of the PC matrix. (b) Average minimum principal strain in the absence and presence of the PC matrix. (c) Representative contour plot of the maximum principal strain in the absence of the PC matrix. (d) Average maximum principal strain in the absence and presence of the PC matrix.

intracellular regions (IC) was found to be significantly smaller than ramp loading $\left(e_{2}=0.0119 \pm 0.0027, p<0.03\right)$. Similarly, the equilibrium PC matrix lateral strain was greater than during ramping, reaching $0.0231 \pm 0.0038$ (Fig. 2).

\section{Study 2: Finite element analysis of cell deformation}

Contour plots of the resulting fluid pressure $(p)$, minimum $\left(e_{1}\right)$ and maximum $\left(e_{2}\right)$ principal strains are reported in Fig. 4 and Fig. 5 for a spherical and ellipsoidal cell, respectively. At peak ramp loading, the intracellular fluid pressure was found to be uniform across the entire IC region and possessed a magnitude that is greater than the $\mathrm{PC}$ and EC matrix pressures (Fig. 4 and Fig. 5). The microenvironments of the spherical and ellipsoidal cells possessed similar fluid pressure in the EC and PC matrices. However, the intracellular pressure in the ellipsoidal cell was found to be $\sim 2 \mathrm{X}$ greater than in the spherical cell. The larger pressure gradient across the membrane of the ellipsoidal cell, therefore, resulted in greater fluid flow in its microenvironment compared to the spherical cell (as indicated by the length of the vectors in Fig. $4 \mathrm{~b}$ vs. Fig. $5 b)$.
Under 2\% platen-to-platen deformation, the minimum principal strain $\left(e_{1}\right)$ at the peak ramp was found to be $0.0193 \pm 0.0024$ in the EC region. This tissue-level deformation resulted in a greater minimum principal strain in the IC region of the chondrocyte, with the ellipsoidal cell being exposed to a slightly greater magnitude of strain $\left(e_{1}:-0.0701 \pm 0.001\right.$ for ellipsoidal vs. $-0.0613 \pm 3 \times 10^{-4}$ for spherical cell). Interestingly, the PC was exposed to a range of compressive strains ranging from a minimum value concentrated near the major pole (apex) of the elongated cell $\left(e_{1}:-0.049 \pm 0.006\right.$; Fig. 5c), and a maximum value occurring near the minor pole (lateral side; $\left.e_{1}:-0.028 \pm 0.002\right)$. A similar pattern of PC compressive strain was seen in the spherical cell, though the range of the PC strain was found to be greater (range in PC matrix strain: -0.03 in spherical cell vs. -0.021 in ellipsoidal cell).

The maximum principal strain $\left(e_{2}\right)$ demonstrated similar pattern in the microenvironments of the spherical and ellipsoidal cells (Fig. 4d and Fig. 5d), with the EC region experiencing an average value of $0.0081 \pm 9 \times 10^{-4}$. The IC region was exposed to a greater magnitude of tensile strain $\left(e_{2}\right)$ than the EC region, reaching a value of $0.033 \pm$ $5 \times 10^{-4}$. Additionally, $e_{2}$ in the PC matrix near the apical 
(major) node was found to be greater in the ellipsoidal cell than in the spherical cell (Fig. 4d vs. Fig. 5d).

The FEA was repeated for an ellipsoidal cell in the absence of the PC matrix, in order to distinguish the contribution of the $\mathrm{PC}$ region. The absence of the $\mathrm{PC}$ matrix did not alter the magnitude of $e_{1}$ or $e_{2}$ in the $\mathrm{EC}$ region of the tissue (Fig. 6). However, the resulting $e_{1}$ in the IC region was found to be significantly smaller in the absence of the PC matrix, $\left(e_{1}:-0.0397 \pm 8 \times 10^{-4}\right.$ without PC vs. $-0.0698 \pm$ $5 \times 10^{-4}$ with PC matrix). Additionally, the magnitude of $e_{2}$ in the IC region was significantly diminished in the absence of the PC matrix (Fig. 6).

\section{Discussion}

The goal of this study was to assess the in situ loading environment of chondrocytes under transient loading using experimental and computational approaches. While previous studies in the literature have reported cell deformation and strains under equilibrium conditions, the current study provides results for the early time response in unconfined compression as well, which are expected to be more representative of physiological loading conditions (Park et al., 2003).

The first observation from Study 1 is that the intracellular strain magnitudes are considerably larger than the applied strain on the EC matrix. In this study, a nominal $2 \%$ platen-to-platen strain was applied to full thickness tissue samples; however, due to depth-dependent inhomogeneity in the compressive mechanical properties of cartilage, this translated into an actual EC matrix compressive strain of $0.47 \%$ on average in the $\mathrm{MZ}$. The diminished magnitude of strain in the MZ may also be due to compliance in the loading device. Consequently, IC and PC strain magnitudes need to be compared to this reference EC matrix value. It was found that the minimum (compressive) principal strain was nearly $7 \mathrm{X}$ higher inside the cell, and $\sim 5 \mathrm{X}$ higher in the $\mathrm{PC}$ region (Fig. 2). A similar strain amplification mechanism was observed in the maximum (tensile) principal strain, with the IC strain nearly $7 \mathrm{X}$ higher than the EC matrix strain, and the PC matrix strain more than $8 \mathrm{X}$ higher (Fig. 2). The experimental results indicate that IC, PC and EC regions behave grossly as incompressible solids during the transient ramping phase, as indicated by the ratio of lateral tensile strain $\left(e_{2}\right)$ to axial compressive strain $\left(e_{1}\right)$ (yielding $\sim 0.5$ in all regions).

This strain amplification persisted even after equilibrium was reached. In the equilibrium response, the EC matrix compressive strain was found to be higher than during the transient ramp $(0.79 \%$ versus $0.47 \%)$, which can also be explained by possible compliance of the loading device: During the transient ramp, the compressive stress acting on the tissue sample is significantly larger than under equilibrium conditions when the stress has relaxed; consequently, a greater amount of deformation gets shifted to the compliant loading device during the transient response, thereby producing a smaller specimen deformation. When normalizing the equilibrium IC and PC principal strains to the corresponding EC matrix strains, it is found that the compressive strain is $6 \mathrm{X}$ greater in the IC and nearly $4 \mathrm{X}$ greater in the PC matrix. A similar amplification was found for the tensile strains as well (Fig. 2).

An axisymmetric finite element analysis was utilized to compare and validate the experimental findings of this study. The results of the FEA demonstrate significant strain amplification mechanism in the IC region (Fig. 4 - Fig. 6 ), greater than had previously been suggested in earlier computational studies of cell-EC matrix interactions (e.g. (Guilak and Mow, 2000)). In the FEA, the applied EC compressive strain is $\sim 2 \%$ and the minimum principal strain in the IC is $7.0 \%$, which represents a magnification of $\sim 3.5 \mathrm{X}$. The patterns of strain distribution in the FEM results also show a reasonable qualitative agreement with experimental results, demonstrating higher values of $e_{1}$ in the IC and lower values on the minor poles of the ellipsoidal cell (Fig. 3 vs. Fig. 5). Similarly, while mostly positive, the maximum principal strain $e_{2}$ exhibits negative values at the major poles of the ellipsoid, both experimentally and theoretically. Given the simplifications inherent in this FEA, such as the assumption that the cell is isolated and that the material properties are homogeneous within the $\mathrm{EC}$ and IC regions, these agreements are encouraging and serve to reinforce the significance of the experimental findings.

The results of this study suggest that cellular geometry affects the local deformation behaviour in and around the cell, as has been previously examined (Baer et al. 2003). Specifically, the ellipsoidal cell was found to possess a greater fluid pressure gradient and larger magnitude of fluid flow as compared to the spherical cell (Fig. 4 vs. Fig. 5). Additionally, the ellipsoidal cell exhibited a higher magnitude of $e_{1}$ in the intracellular region than the spherical cell. It is interesting to note that the magnitude of strain in the PC region was more uniform around an ellipsoidal cell than a spherical cell, suggesting that the shape of the cell may influence the behaviour of the surrounding tissues, not solely modulating the cell's own mechanical behaviour (Fig. 4 vs. Fig. 5).

In order to compare the contribution of the PC matrix alone versus the effect of the cell geometry, the FEA analysis was repeated in the absence of a PC matrix for an ellipsoidal cell. The results indicate that the presence of PC matrix significantly increases the magnitude of $e_{1}$ and $e_{2}$ in the IC region (Fig. 6). The strain amplification in $e_{1}$ due to the presence of a PC matrix is $\sim 3.5$, in the IC region normalized to the EC strain. This strain amplification is consistent with previously reported values (Alexopoulos et al., 2005a). Interestingly, the absence of a PC matrix resulted in a decrease in the IC strain relative to the EC strain, though it did not cause the strain amplification behaviour to disappear entirely (Fig. 6). Instead, the strain amplification in the absence of the PC matrix was found to be $\sim 2 \mathrm{X}$ (compared to $3.5 \mathrm{X}$ in presence of $\mathrm{PC}$ matrix). The existence of strain amplification with no PC matrix may be due to the (1) differences in the Young's modulus of the cell and EC matrix, and (2) the low permeability of the cell membrane, preventing fluid from escaping the cell during loading. 
Based on the finite element analysis, this strain amplification is understood to result from the large disparity between EC matrix and intracellular properties. The low permeability of the cell membrane restricts water outflow or inflow into the cell within physiological time scales; consequently the cell behaves as a nearlyincompressible soft gel within a much stiffer environment. These results suggest that varying regions of the cell, such as the nucleus or cytoskeletal elements, may be exposed to varying magnitudes of strain, based on the spatial variation seen in the cell and its microenvironment.

Biomechanical studies are always representative of a certain scale in the hierarchy from the organ level to the molecular level. This study analyzes the intermediate scale between the tissue and cellular levels, not between the cellular and molecular levels. Unlike a recent study (Knight et al., 2006), neither the experimental results nor the finite element analysis resolve the strain field at the level of the heterogeneous intracellular structures, such as organelles or cytoskeletal elements. The current results analyze the in situ response of chondrocytes in their native cartilage environment, under physiological time scales, providing novel insight into a mechanism of strain amplification with important implications for mechanotransduction. We postulate that mechanotransduction may be mediated by this amplification mechanism, such that the elevated magnitudes of strain in the intracellular regions may alter the biosynthetic pathways of the cell when loaded in situ, possibly by altering the conformation of molecular structures in the nucleus and cytoplasm, which in turn may alter transcription, translation or post-translational modifications.

From the results of this study, it appears that the PC matrix is acting as a mechanical transducer for the cell, by propagating greater magnitudes of strain to the IC compared to the $\mathrm{PC}$ or EC regions. These mechanical signals may play a role in altering the response of the cell to interactions with hyaluronan or type VI collagen found in the PC matrix. Additionally, the results suggest that studies examining the chondrocyte's response to loading and injury may be more appropriately interpreted in the context of the strains translated to the cell and its microenvironment, not solely by the applied tissue loading magnitude.

In summary, the current study provides results for the early time response of chondrocyte deformation in situ, which are expected to be more representative of physiological loading conditions. We conclude that EC matrix strains get magnified very significantly within and around the cellular environment during transient loading and at equilibrium.

\section{Acknowledgements}

This study was supported by NIH (NIAMS) AR46532 and AR46568.

\section{References}

Akizuki S, Mow VC, Muller F, Pita JC, Howell DS, Manicourt DH (1986) Tensile properties of human knee joint cartilage: I. Influence of ionic conditions, weight bearing, and fibrillation on the tensile modulus. J Orthop Res 4: 379-392.

Alexopoulos LG, Setton LA, Guilak F (2005a) The biomechanical role of the chondrocyte pericellular matrix in articular cartilage. Acta Biomater 1: 317-325.

Alexopoulos LG, Williams GM, Upton ML, Setton LA, Guilak F (2005b) Osteoarthritic changes in the biphasic mechanical properties of the chondrocyte pericellular matrix in articular cartilage. J Biomech 38: 509-517.

Almeida ES, Spilker RL (1997) Mixed and penalty finite element models for the nonlinear behavior of biphasic soft tissues in tinite deformation: Part I - Alternate formulations. Comput Methods Biomech Biomed Engin 1: $25-46$.

Ateshian GA, Costa KD, Hung CT (2007) A theoretical analysis of water transport through chondrocytes. Biomech Model Mechanobiol 6: 91-101.

Baer AE, Laursen TA, Guilak F, Setton LA (2003) The micromechanical environment of intervertebral disc cells determined by a finite deformation, anisotropic, and biphasic finite element model. J Biomech Eng 125: 1-11.

Buschmann MD, Kim YJ, Wong M, Frank E, Hunziker EB, Grodzinsky AJ (1999) Stimulation of aggrecan synthesis in cartilage explants by cyclic loading is localized to regions of high interstitial fluid flow. Arch Biochem Biophys 366: 1-7.

Chahine NO, Wang CC, Hung CT, Ateshian GA (2004) Anisotropic strain-dependent material properties of bovine articular cartilage in the transitional range from tension to compression. J Biomech 37: 1251-1261.

Clark AL, Barclay LD, Matyas JR, Herzog W (2003) In situ chondrocyte deformation with physiological compression of the feline patellofemoral joint. J Biomech 36: 553-568.

Gray ML, Pizzanelli AM, Grodzinsky AJ, Lee RC (1988) Mechanical and physiochemical determinants of the chondrocyte biosynthetic response. J Orthop Res 6: 777-792.

Guilak F (1995) Compression-induced changes in the shape and volume of the chondrocyte nucleus. J Biomech 28: 1529-1541.

Guilak F (2000) The deformation behavior and viscoelastic properties of chondrocytes in articular cartilage. Biorheology 37: 27-44.

Guilak F, Mow VC (2000) The mechanical environment of the chondrocyte: a biphasic finite element model of cell-matrix interactions in articular cartilage. J Biomech 33: 1663-1673.

Guilak F, Meyer BC, Ratcliffe A, Mow VC (1994) The effects of matrix compression on proteoglycan metabolism in articular cartilage explants. Osteoarthritis Cartilage 2: 91-101.

Guilak F, Ratcliffe A, Mow VC (1995) Chondrocyte deformation and local tissue strain in articular cartilage: a confocal microscopy study. J Orthop Res 13: 410-421. 
Guilak F, Sah RL, Setton LA (1997) Physical regulation of cartilage metabolism. In: Basic Orthopaedic Biomechanics. Mow VC, Hayes WC (eds). LippincottRaven, Philadelphia. pp 179-207.

Guilak F, Jones WR, Ting-Beall HP, Lee GM (1999). The deformation behavior and mechanical properties of chondrocytes in articular cartilage. Osteoarthritis Cartilage 7: 59-70.

Guilak F, Tedrow JR, Burgkart R (2000) Viscoelastic properties of the cell nucleus. Biochem Biophys Res Commun 269: 781-786.

Guilak, F, Erickson GR, Ting-Beall HP (2002). The effects of osmotic stress on the viscoelastic and physical properties of articular chondrocytes. Biophys J 82: 720727.

Kempson GE, Freeman MA, Swanson SA (1968) Tensile properties of articular cartilage. Nature 220: $1127-$ 1128.

Kim YJ, Sah RL, Grodzinsky AJ, Plaas AH, Sandy JD (1994) Mechanical regulation of cartilage biosynthetic behavior: physical stimuli. Arch Biochem Biophys 311: $1-12$.

Knight MM., Ghori SA, Lee DA, Bader DL (1998) Measurement of the deformation of isolated chondrocytes in agarose subjected to cyclic compression. Med Eng Phys 20: 684-688.

Knight MM, Van de Breevaart Bravenboer J, Lee DA, Van Osch GJ, Weinans H, Bader DL (2002) Cell and nucleus deformation in compressed chondrocyte-alginate constructs: temporal changes and calculation of cell modulus. Biochim Biophys Acta 1570: 1-8.

Knight MM, Bomzon Z, Kimmel E, Sharma AM, Lee DA, Bader DL (2006) Chondrocyte deformation induces mitochondrial distortion and heterogeneous intracellular strain fields. Biomech Model Mechanobiol 5: 180-191

Krishnan R, Park S, Eckstein F, Ateshian GA (2003) Inhomogeneous cartilage properties enhance superficial interstitial fluid support and frictional properties, but do not provide a homogeneous state of stress. J Biomech Eng 125: 569-577.

Lee DA, Bader DL (1995) The development and characterization of an in vitro system to study straininduced cell deformation in isolated chondrocytes. In Vitro Cell Dev Biol Anim 31: 828-835.

Maroudas A (1979) Physico-chemical properties of articular cartilage. In: Adult articular cartilage. Freeman MAR (ed). Pitman Medical, Tunbridge Wells. pp. 215290.

Maroudas A, Bannon C (1981). Measurement of swelling pressure in cartilage and comparison with the osmotic pressure of constituent proteoglycans. Biorheology 189: 619-632.

Mow VC, Kuei SC, Lai WM, Armstrong CG (1980) Biphasic creep and stress relaxation of articular cartilage in compression? Theory and experiments. J Biomech Eng 102: 73-84.

Palmoski M, Perricone E, Brandt KD (1979) Development and reversal of a proteoglycan aggregation defect in normal canine knee cartilage after immobilization. Arthritis Rheum 22: 508-517.
Palmoski MJ, Brandt KD (1984) Effects of static and cyclic compressive loading on articular cartilage plugs in vitro. Arthritis Rheum 27: 675-681.

Park S, Krishnan R, Nicoll SB, Ateshian GA (2003) Cartilage interstitial fluid load support in unconfined compression. J Biomech 36: 1785-1796.

Sah RL, Doong JY, Grodzinsky AJ, Plaas AH, Sandy JD (1991) Effects of compression on the loss of newly synthesized proteoglycans and proteins from cartilage explants. Arch Biochem Biophys 286: 20-29.

Sah RL, Kim YJ, Doong JY, Grodzinsky AJ, Plaas AH, Sandy JD (1989) Biosynthetic response of cartilage explants to dynamic compression. J Orthop Res 7: 619636.

Schinagl RM, Gurskis D, Chen AC, Sah RL (1997) Depth-dependent confined compression modulus of fullthickness bovine articular cartilage. J Orthop Res 15: 499506.

Schinagl RM, Ting MK, Price JH, Sah RL (1996) Video microscopy to quantitate the inhomogeneous equilibrium strain within articular cartilage during confined compression. Ann Biomed Eng 24: 500-512.

Setton LA, Mow VC, Howell DS (1995) Mechanical behavior of articular cartilage in shear is altered by transection of the anterior cruciate ligament. J Orthop Res 13: 473-482.

Soltz MA, Ateshian GA (2000) A conewise linear elasticity mixture model for the analysis of tensioncompression nonlinearity in articular cartilage. J Biomech Eng 122: 576-586.

Trickey WR, Lee GM, Guilak F (2000) Viscoelastic properties of chondrocytes from normal and osteoarthritic human cartilage. J Orthop Res 18: 891-898.

Trickey WR, Baaijens FP, Laursen TA, Alexopoulos LG, Guilak F (2006) Determination of the Poisson's ratio of the cell: recovery properties of chondrocytes after release from complete micropipette aspiration. J Biomech 39: $78-87$.

Valhmu WB, Stazzone EJ, Bachrach NM, Saed-Nejad F, Fischer SG, Mow VC, Ratcliffe A (1998) Loadcontrolled compression of articular cartilage induces a transient stimulation of aggrecan gene expression. Arch Biochem Biophys 353: 29-36.

Wang CC, Hung CT, Mow VC (2001) An analysis of the effects of depth-dependent aggregate modulus on articular cartilage stress-relaxation behavior in compression. J Biomech 34: 75-84.

Wang CC, Deng JM, Ateshian GA, Hung CT (2002a) An automated approach for direct measurement of twodimensional strain distributions within articular cartilage under unconfined compression. J Biomech Eng 124: $557-$ 567.

Wang CC, Guo XE, Sun D, Mow VC, Ateshian GA, Hung CT (2002b) The functional environment of chondrocytes within cartilage subjected to compressive loading: a theoretical and experimental approach. Biorheology 39: 11-25.

Wang CC, Chahine NO, Hung CT, Ateshian GA (2003) Optical determination of anisotropic material properties of bovine articular cartilage in compression. J Biomech 36: 339-353. 
Wayne JS, Woo SL, Kwan MK (1991) Application of the u-p finite element method to the study of articular cartilage. J Biomech Eng 113: 397-403.

Wong M, Siegrist M, Cao X (1999) Cyclic compression of articular cartilage explants is associated with progressive consolidation and altered expression pattern of extracellular matrix proteins. Matrix Biol 18: 391-399.

Woo SL, Akeson WH, Jemmott GF (1976) Measurements of nonhomogeneous, directional mechanical properties of articular cartilage in tension. $\mathrm{J}$ Biomech 9: 785-791.

Wu JZ, Herzog W (2000) Finite element simulation of location- and time-dependent mechanical behavior of chondrocytes in unconfined compression tests. Ann Biomed Eng 28: 318-330.

Wu JZ, Herzog W, Epstein M (1999) Modelling of location- and time-dependent deformation of chondrocytes during cartilage loading. J Biomech 32: 563-572.

Xu X, Cui Z, Urban JP (2003) Measurement of the chondrocyte membrane permeability to Me2SO, glycerol and 1,2-propanediol. Med Eng Phys 25: 573-579.

Youn I, Choi JB, Cao L, Setton LA, Guilak F (2006) Zonal variations in the three-dimensional morphology of the chondron measured in situ using confocal microscopy. Osteoarthritis Cartilage 14: 889-897.

\section{Discussion with Reviewers}

C.W.A. Archer: What are the implications of these findings in relation to what we know about the pericellular biology of mid-zone chondrocytes?

Authors: The results of this study highlight the impact that the pericellular (PC) matrix plays in regulating stress/ strain and fluid flow in the chondrocyte, similar to previous studies in the literature (Alexopoulos et al., 2005a). Our findings indicate that the presence of the PC matrix significantly increases the magnitude of minimum and maximum principal strains in the intracellular (IC) region, compared to the EC matrix. Additionally, the PC matrix appears to be exposed to strain amplification relative to the EC matrix, though these magnitudes are smaller than seen in the IC regions. These results indicate that the PC matrix is acting as a mechanical transducer for the cell, by propagating greater magnitudes of strain to the IC compared to the PC or EC regions. These mechanical signals may play a role in altering the response of the cell to interactions with hyaluronan or type VI collagen found in the PC matrix.

C.W.A. Archer: Is it correct to model the cell as a 'homogenous gel' as clearly structural components such as the cytoskeleton especially intermediate filaments and other elements compartmentalise the whole structure?

Authors: Biomechanical studies are always representative of a certain scale in the hierarchy from the organ level to the molecular level. This study analyzes the intermediate scale between the tissue and cellular levels, not between the cellular and molecular levels. The current results analyze the in situ response of chondrocytes in their native cartilage environment, under physiological time scales, providing novel insight into a mechanism of strain amplification between the extracellular matrix and the cell proper, with important implications for mechanotransduction. How these strains are transduced to subcellular structures may be addressed in future studies.

B. Sah: There is a mention of the quality of strain analysis. It will be useful to know how this method was validated and what the accuracy is, considering the very small extracellular matrix strains $<1 \%$. The accuracy of this technique is important to the interpretation of the findings. Authors: We agree with the reviewer that the quality of the strain analysis is pivotal in the interpretation of the findings. Prior to running these experiments, we had undertaken an enormous effort to validate this technique. These efforts included imaging techniques to improve the quality of the images and resulting texture in cell and extracellular matrix. Additionally, in choosing the subset size, step size and smoothing window size used in the DICan iterative method was used to minimize the error potentially introduced by the choice of these parameters. In presenting some of this validation work, we have added a table that summarizes some of the relevant measures (Table 1). The results indicate that accuracy of the strain analysis increases with increasing deformation. At the smallest applied deformation of $0.5 \%$ strain, the DIC technique has an accuracy of $\sim 10 \%$, which we believe to be reasonable and acceptable. 